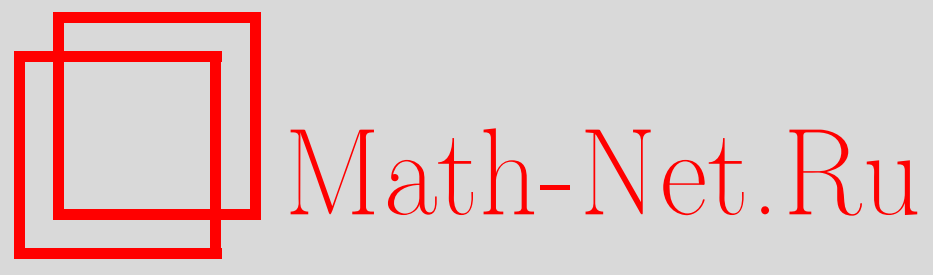

K.-H. Indlekofer, О. И. Клесов, Усиленный закон больших чисел для кратных сумм, индексы которых принадлежат сектору, ограниченному двумя функциями, Теория вероятн. и ее примен., 2007, том 52, выпуск 4, 803-810

DOI: https://doi.org/10.4213/tvp1649

Использование Общероссийского математического портала MathNet.Ru подразумевает, что вы прочитали и согласны с пользовательским соглашением

http: //www . mathnet.ru/rus/agreement

Параметры загрузки:

IP : 54.81 .137 .203

26 апреля 2023 г., 11:41:33

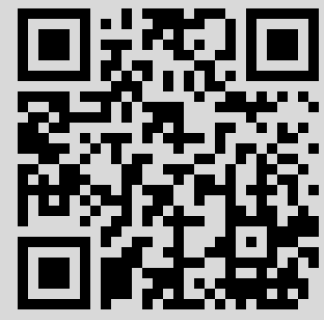


(C) 2007

ИНДЛЕКОФЕР К.-Х.*, КЛЕСОВ О. И.**

\section{УСИЛЕННЫЙ ЗАКОН БОЛЬШИХ ЧИСЕЛ ДЛЯ КРАТНЫХ СУММ, ИНДЕКСЫ КОТОРЫХ ПРИНАДЛЕЖАТ СЕКТОРУ, ОГРАНИЧЕННОМУ ДВУМЯ ФУНКЦИЯМИ ${ }^{1)}$}

Найдены необходимые и достаточные условия справедливости усиленного закона больших чисел для двойных сумм независимых одинаково распределенных случайных величин для случая, когда индексы сумм принадлежат сектору, граница которого образована двумя функциями.

Ключевые слова и фразы: усиленный закон больших чисел, двойные суммы, случайные поля.

1. Введение. Пусть $d \geqslant 1$ и $\mathrm{N}^{d}$ - пространство $d$-мерных векторов с целыми положительными координатами. Рассмотрим семейство $\left\{X\left(k_{1}, \ldots, k_{d}\right)\right.$; $\left.k_{1} \geqslant 1, \ldots, k_{d} \geqslant 1\right\}$ независимых в совокупности одинаково распределенных случайных величин. Для $n_{1} \geqslant 1, \ldots, n_{d} \geqslant 1$ положим

$$
S\left(n_{1}, \ldots, n_{d}\right)=\sum_{k_{1}=1}^{n_{1}} \cdots \sum_{k_{d}=1}^{n_{d}} X\left(k_{1}, \ldots, k_{d}\right) .
$$

Целью этой работы является изучение поведения почти наверное «прямоугольных» сумм $S\left(n_{1}, \ldots, n_{d}\right)$ при изменении их индексов в определенном множестве. Аналогом такой постановки в классическом случае $d=1$ является усиленный закон больших чисел для подпоследовательностей. Основным результатом работы является теорема 1. В п. 3 мы приводим также несколько ее следствий для конкретных случаев.

Закон больших чисел для кратных сумм независимых одинаково распределенных случайных величин получен в работе Р. Т. Смайза [6] для случая, когда индексы меняются без ограничений. В [6] доказано, что соотношение

$$
\mathbf{P}\left\{\left|\frac{S\left(n_{1}, \ldots, n_{d}\right)}{n_{1} \cdots n_{d}}-\mu\right| \geqslant \varepsilon \text { б.ч. }\right\}=0 \quad \text { для всех } \varepsilon>0
$$

выполняется для некоторой неслучайной константы $\mu$ тогда и только тогда, когда

$$
\mathbf{E} X=\mu, \quad \mathrm{E}|X|\left(\ln ^{+}|X|\right)^{d-1}<\infty .
$$

Здесь и в дальнейшем аббревиатура «б.ч.» означает «бесконечное количество раз» (или «бесконечно часто»), $X$ - это случайная величина, имеющая то же распределение, что и все другие случайные величины $X\left(k_{1}, \ldots, k_{d}\right), \ln ^{+} z=\ln (1+z)$ для $z \geqslant 0$. Этот результат совпадает с классическим законом больших чисел Колмогорова в случае $d=1$, но при $d>1$ соотношение (1.2) формально сильнее, чем утверждение о том, что семейство случайных величин $\left\{S\left(n_{1}, \ldots, n_{d}\right) /\left(n_{1} \cdots n_{d}\right)\right\}$ имеет предел почти наверное при $n_{1} \rightarrow \infty, \ldots, n_{d} \rightarrow \infty$.

В определенном смысле можно считать, что исследования соотношений, подобных (1.2), были начаты Н. Винером [7], который изучал эргодическую теорему для некоммутативных групп преобразований, сохраняющих меру в измеримом пространстве. Винер [7] рассматривал случай кратных интегралов, а не сумм, причем областью интегрирования у него были расширяющиеся шары.

Результат работы [7] обобщен А. Зигмундом [8], который также изучал непрерывный случай в эргодической теореме, хотя областью интегрирования у него были

* Fachbereich Mathematik-Informatik, Universität Paderborn, Warburger Str., 100, 33098 Paderborn, Germany; e-mail: k-heinz@uni-paderborn.de

** Кафедра математического анализа и теории вероятностей, Национальный технический университет Украины, пр-т Победы, 37, 02056 Киев, Украина; e-mail: tbimc@ln.ua

1) Работа выполнена при поддержке грантом DFG 436 UKR 113/41/0-2. 
параллелепипеды вместо шаров. Н. Данфорд [2] одновременно и независимо от Зигмунда [8] изучал эргодическую теорему для дискретного времени, причем вместо интегралов он рассматривал кратные суммы. Постановка задачи в [2] наиболее близка к нашей.

А. Гут [3] первым рассмотрел усиленный закон больших чисел для кратных сумм, индексы которых удовлетворяют некоторым ограничениям.

$\mathrm{O}$ п р е д е л е н и е. Пусть заданы бесконечное подмножество $A \subseteq \mathbf{N}^{d}$ и семейство (поле) действительных чисел $\left\{c\left(k_{1}, \ldots, k_{d}\right) ; k_{1} \geqslant 1, \ldots, k_{d} \geqslant 1\right\}$. Мы пишем

$$
\lim _{A} c\left(n_{1}, \ldots, n_{d}\right)=c
$$

если для любого $\varepsilon>0$ неравенство $\left|c\left(n_{1}, \ldots, n_{d}\right)-c\right|<\varepsilon$ выполняется для всех векторов $\left(n_{1}, \ldots, n_{d}\right) \in A$, кроме, возможно, конечного их числа.

Определим также последовательность Дирихле $\left\{\tau_{n}, n \geqslant 1\right\}$ на множестве $A$ следуюшим образом:

$$
\tau_{n}=\operatorname{card}\left\{\left(k_{1}, \ldots, k_{d}\right) \in A: k_{1} \cdots k_{d}=n\right\}, \quad n \geqslant 1 .
$$

$\mathrm{B}$ [3] изучен следующий вопрос: какие условия на распределение случайных величин $X\left(k_{1}, \ldots, k_{d}\right)$ являются необходимыми и достаточными в случае $A=A_{\theta}$ для того, чтобы

$$
\lim _{A} \frac{S\left(n_{1}, \ldots, n_{d}\right)}{n_{1} \cdots n_{d}}=\mu \quad \text { п.н., }
$$

где $A=A_{\theta}=\left\{\left(n_{1}, \ldots, n_{d}\right): \theta n_{i} \leqslant n_{j} \leqslant \theta^{-1} n_{i}\right.$ для всех $\left.1 \leqslant i<j \leqslant d\right\}$, а $0<\theta<1$ фиксировано? Оказалось, что ответ не зависит от $\theta$ и таким условием является выполнение равенства

$$
\mathbf{E} X=\mu .
$$

Вполне естественным является желание обобщить постановку Гута на случай других множеств $A$ вместо секторов $A_{\theta}$. Подобная попытка предпринята в статье [5] для случая $d=2$ и $A=\left\{(i, j): g_{1}(i) \leqslant j \leqslant g_{2}(i)\right\}$, где возрастающие функции $g_{1}$ и $g_{2}$ таковы, что

$$
\begin{gathered}
g_{1}(x) \leqslant x \leqslant g_{2}(x) \quad \text { для всех } x \geqslant 1, \\
\frac{g_{1}(x)}{x} \downarrow \quad \text { и } \quad \frac{g_{2}(x)}{x} \uparrow .
\end{gathered}
$$

Случай, рассмотренный в [3], отвечает выбору функций $g_{1}(x)=\theta x$ и $g_{2}(x)=\theta^{-1} x$.

Достаточное условие для усиленного закона больших чисел на $A$ в работе [5] имеет следующий вид:

$$
\sum_{(i, j) \in A} \mathbf{P}\{|X| \geqslant i j\}<\infty,
$$

где $X$ - копия случайной величины $X(1,1)$. Необходимость условия $(1.8)$ не обсуждалась в [5].

Целью этой заметки является изучение усиленного закона больших чисел для случая $d=2$ при отказе от условия (1.7). При этом мы доказываем, что условия являются необходимыми и достаточными. В доказательстве применяется новый метод, не требующий подсчета количества целых точек в области $A$, но зависящий от некоторых свойств последовательности $T_{n}=\tau_{1}+\cdots+\tau_{n}$, которые мы устанавливаем без использования асимптотических формул для $T_{n}$. Идея заключается в том, чтобы оперировать непосредственно с двойными рядами, не сводя их к однократным (когда и возникает необходимость вычисления $T_{n}$ ). Этот метод требует больше технических преобразований, но в конце концов дает лучший результат, чем в [5].

2. Основной результат. В дальнейшем мы ограничиваемся случаем $d=2$. Рассмотрим неубывающую функцию $f$ такую, что

$$
f(x) \geqslant x \quad \text { для всех } x \geqslant 1 .
$$

Определим множество $A$ следуюшим образом:

$$
A=\left\{(i, j) \in \mathbf{N}^{2}: i \leqslant j \leqslant f(i)\right\} .
$$


Рассмотрим поле независимых одинаково распределенных случайных величин $\left\{X(i, j) ;(i, j) \in \mathbf{N}^{2}\right\}$ и поле их прямоугольных сумм $\left\{S(m, n) ;(m, n) \in \mathbf{N}^{2}\right\}$ :

$$
S(m, n)=\sum_{i=1}^{m} \sum_{j=1}^{n} X(i, j) .
$$

Пусть $X$ - это копия случайной величины $X(1,1)$. Функцию распределения случайной величины $X$ обозначим $F$.

Теорема 1. Пусть неубывающая функиия $f$ удовлетворяет условию (2.1), а множество $A$ определено в (2.2). Усиленный закон больиих иисел

$$
\lim _{A} \frac{S(m, n)}{m n}=\mu \quad \text { n.н., }
$$

где $\mu$ - некоторая неслучайная константа, эквивалентен совокупности условий (1.5) $u(1.8)$.

3 а м е ч а н и е 1 . Пусть $\tau_{k}, k \geqslant 1$, - это количество решений уравнения $i j=k$ таких, что $(i, j) \in A$. Условие (1.8) можно переписать в виде

$$
\sum_{k=1}^{\infty} \tau_{k} \mathbf{P}\{|X| \geqslant k\}<\infty .
$$

Это условие равносильно следуюшему:

$$
\sum_{k=1}^{\infty} T_{k} \mathbf{P}\{k \leqslant|X|<k+1\}<\infty,
$$

где $T_{1}=\tau_{1}$ и $T_{k}=\tau_{1}+\cdots+\tau_{k}, k \geqslant 2$. Условие (1.8) вытекает из того, что $\mathbf{E} T(|X|)<\infty$, где $T(x)=T_{[x]}$. Если $T$ изменяется не слишком быстро в том смысле, что $T(x+1)=$ $O(T(x))$, то последнее условие эквивалентно (1.8). Подобными рассуждениями мы воспользуемся в п. 3.

Для доказательства теоремы 1 нам необходимы два вспомогательных результата. Положим $\Pi(m, n)=\{(i, j): 1 \leqslant i \leqslant m, 1 \leqslant j \leqslant n\}$ для $m \geqslant 1$ и $n \geqslant 1$. Мы говорим, что семейство $\mathscr{A}$ множеств $A_{\alpha}$ линейно упорядочено (по отношению к включению множеств), если для каждой пары $A_{\alpha}, A_{\beta} \in \mathscr{A}$ выполнено по крайней мере одно из двух включений: $A_{\alpha} \subseteq A_{\beta}$ или $A_{\beta} \subseteq A_{\alpha}$.

Лемма 1. Пусть

$$
B=\left\{(i, j) \in \mathbf{N}^{2}: j>f(i)\right\}, \quad C=\left\{(i, j) \in \mathbf{N}^{2}: j<i\right\} .
$$

Оба семейства множеств $\{B \cap \Pi(m, n) ;(m, n) \in A\}$ u $\{C \cap \Pi(m, n) ;(m, n) \in A\}$ являются линейно упорядоченными.

Аналогично, мы говорим, что семейство $\mathscr{V}$ точек $\left(m_{\alpha}, n_{\alpha}\right)$ линейно упорядочено (покоординатно), если для каждой пары $\left(m_{\alpha}, n_{\alpha}\right),\left(m_{\beta}, n_{\beta}\right) \in \mathscr{V}$ выполнено по крайней мере одно из условий: а) $m_{\alpha} \leqslant m_{\beta}$ и $n_{\alpha} \leqslant n_{\beta}$, b) $m_{\beta} \leqslant m_{\alpha}$ и $n_{\beta} \leqslant n_{\alpha}$.

Лемма 2. Пусть внутренность $и$ гранича произвольного множества $D \in \mathrm{N}^{2}$ определены следуюиим образом:

$\operatorname{int}(D)=\{(i, j) \in D:(i-1, j) \in D,(i, j-1) \in D,(i-1, j-1) \in D\}, \quad D=D \backslash \operatorname{int}(D)$.

Положим

$$
B=\left\{(i, j) \in \mathbf{N}^{2}: j \geqslant f(i)\right\} \quad \text { u } C=\left\{(i, j) \in \mathbf{N}^{2}: j \leqslant i\right\} .
$$

Оба семейства точек $\partial(B)$ и $\partial(C)$ являются линейно упорядоченньми.

Д о к а 3 а т е л ь с т в о л е м м ы 1. Доказательство проведем только для семейства $\{B \cap \Pi(m, n) ;(m, n) \in A\}$. Доказательство для $\{C \cap \Pi(m, n) ;(m, n) \in A\}$ совершенно аналогично. Для $n \geqslant 1$ положим $m^{*}=m^{*}(n)=\min \{m: f(m) \geqslant n\}$. Заметим, что поскольку $m^{*} \leqslant m$ и $\left(m^{*}+k, j\right) \notin B$ для всех $k \geqslant 1$ и $j \leqslant n$, то $B \cap \Pi(m, n)=B \cap \Pi\left(m^{*}, n\right)$ для всех $(m, n) \in A$. Пусть теперь $\left(m_{1}, n_{1}\right)$ и $\left(m_{2}, n_{2}\right)-$ два элемента из $A$, а $m_{1}^{*}=m_{1}^{*}\left(n_{1}\right)$ и $m_{2}^{*}=m_{2}^{*}\left(n_{2}\right)$ определены как выше для $n_{1}$ и $n_{2}$ соответственно. Положим $B_{i}=B \cap \Pi\left(m_{i}, n_{i}\right)$ для $i=1,2$. Докажем, что если $n_{2} \leqslant n_{1}$, то $B_{2} \subseteq B_{1}$. Если это не так, то существует точка $(i, j) \in B_{2}$, для которой $(i, j) \notin B_{1}$, т.e.

$$
i \leqslant m_{2}^{*}, \quad j \leqslant n_{2}, \quad j>f(i) \quad \text { и } \quad i>m_{1}^{*} \text { или } j>n_{1} .
$$


Неравенство $j>n_{1}$ противоречит неравенству $j \leqslant n_{2}$ и поэтому не может выполняться. В свою очередь, неравенство $i>m_{1}^{*}$ также не может выполняться, так как в этом случае в силу монотонности функции $f$ было бы выполнено неравенство $f(i) \geqslant f\left(m_{1}^{*}\right)$. Отсюда и из определения $m_{1}^{*}$ вытекало бы, что $j>f(i) \geqslant f\left(m_{1}^{*}\right)>$ $n_{1} \geqslant n_{2}$ и, следовательно, $j>n_{2}$. Это противоречие и доказывает включение $B_{2} \subseteq B_{1}$. Лемма 1 доказана.

Д о ка за те л ь т в о л е м ы 2. Доказательство проведем для $\partial(B)$. Доказательство для $\partial(C)$ совершенно аналогично. Заметим, что

$$
\text { для всех }(i, j) \in B \text { выполнено включение }(i-1, j) \in B \text {. }
$$

Действительно, если бы утверждение (2.4) не выполнялось пля некоторой точки $(i, j) \in B$, мы бы получили $j<f(i-1) \leqslant f(i)$ и, значит, $(i, j) \notin B$. Кроме того,

для всех $(i, j) \in \partial(B)$ имеет место $(i, j-1) \notin B$.

Если бы утверждение (2.5) не выполнялось для некоторой точки $(i, j) \in \partial(B)$, то было бы выполнено включение $(i, j-1) \in B$. Применяя утверждение $(2.4)$, доказываем, что $(i-1, j-1) \in B$, откуда $(i, j),(i-1, j),(i, j-1),(i-1, j-1) \in B$, а это противоречит включению $(i, j) \in \partial(B)$.

Пусть теперь $\left(m_{1}, n_{1}\right)$ и $\left(m_{2}, n_{2}\right)$ - два элемента границы $\partial(B)$. Не теряя общности, предположим, что $m_{1} \leqslant m_{2}$. Докажем, что в этом случае $n_{1} \leqslant n_{2}$. Если бы выполнялось обратное, т.е. $n_{1}>n_{2}$, мы бы имели $n_{2} \leqslant n_{1}-1<f\left(m_{1}\right) \leqslant f\left(m_{2}\right)$, что противоречит включению $\left(m_{2}, n_{2}\right) \in B$. Второе неравенство в этой цепочке вытекает из $\left(m_{1}, n_{1}-1\right) \notin B$, что в свою очередь следует из (2.5). Лемма 2 доказана.

Доказа те ль с т в о т е о е мы 1. Необходимость. Пусть выполнен усиленный закон больших чисел (2.3). Поскольку $(n, n) \in A$ для всех $n \geqslant 1$, то $S(n, n) / n^{2} \rightarrow \mu$ п.н. при $n \rightarrow \infty$. Последовательность $\{S(n, n), n \geqslant 1\}$ можно рассматривать как подпоследовательность $\left\{S_{n_{k}}\right\}$ с $n_{k}=k^{2}$ последовательности сумм $\left\{S_{n}, n \geqslant 1\right\}$ независимых одинаково распределенных случайных величин $\left\{X_{k}, k \geqslant 1\right\}$ с функцией распределения $F$. Следовательно $\left(S_{n_{k+1}}-S_{n_{k}}\right) / n_{k} \rightarrow 0$ п.н. при $k \rightarrow \infty$ и поэтому из леммы Бореля-Кантелли вытекает, что для всех $\varepsilon>0$

$$
\sum_{k=1}^{\infty} \mathbf{P}\left\{\left|S_{2 k+1}\right| \geqslant \varepsilon k^{2}\right\}<\infty .
$$

Поскольку случайные величины $\left\{X_{n}\right\}$ независимы, одинаково распределены и

$$
\left\{\omega:\left|S_{2 k+1}\right| \geqslant \varepsilon k^{2}\right\} \supseteq\left\{\omega:\left|S_{2 k}\right| \geqslant 2 \varepsilon k^{2}\right\} \cap\left\{\omega:\left|X_{2 k+1}\right|<\varepsilon k^{2}\right\},
$$

мы заключаем из (2.6), что $\sum_{k=1}^{\infty} \mathbf{P}\left\{\left|S_{2 k}\right| \geqslant \varepsilon k^{2}\right\}<\infty$ для всех $\varepsilon>0$, откуда следует, что

$$
\sum_{k=1}^{\infty} \mathbf{P}\left\{\left|S_{k}\right| \geqslant \varepsilon k^{2}\right\}<\infty \quad \text { для всех } \varepsilon>0 .
$$

Теперь мы применяем один из результатов работы [1] и доказываем, что $\mathbf{E}|X|<\infty$. Согласно усиленному закону больших чисел Колмогорова, это означает, что $S_{n} / n \rightarrow$ $\mathbf{E} X$ п.н. при $n \rightarrow \infty$. В частности, $S_{n_{k}} / n_{k} \rightarrow \mathbf{E} X$ п.н., значит, $\mu=\mathbf{E} X$. Таким образом, (1.5) доказано.

Остается доказать (1.8). Ясно, что

$$
X(i, j)=S(i, j)-S(i-1, j)-S(i, j-1)+S(i-1, j-1),
$$

значит, из усиленного закона больших чисел (2.3) вытекает, что п.н. неравенство $|X(i, j)| \geqslant i j$ может выполняться только для конечного числа пар $(m, n) \in \operatorname{int}(A)$. Применяя лемму Бореля-Кантелли для независимых событий $\{\omega:|X(i, j)| \geqslant i j\}$, получаем

$$
\sum_{(i, j) \in \operatorname{int}(A)} \mathbf{P}\{|X| \geqslant i j\}<\infty .
$$

Пусть теперь $B$ и $C$ определены как в лемме 2. Понятно, что $\partial(A)=\partial(B) \cup \partial(C)$. Более того, $\partial(C)=\{(i, j): i=j\}$ и поэтому

$$
\sum_{(i, j) \in \partial(C)} \mathbf{P}\{|X| \geqslant i j\}<\infty
$$


в силу условия (1.5) (заметим, что (2.8) равносильно $\mathbf{E}|X|^{1 / 2}<\infty$ ). Семейство точек $\partial(B)$ линейно упорядочено в силу леммы 2 , поэтому уравнение $i j=k$ имеет не более одного решения $(i, j) \in \partial(B)$. Значит, из условия $(1.5)$ вытекает

$$
\sum_{(i, j) \in \partial(B)} \mathbf{P}\{|X| \geqslant i j\}<\infty
$$

Объединяя (2.7)-(2.9), доказываем (1.8).

Достаточность. Пусть условия (1.5) и (1.8) выполнены. Не теряя общности, предположим, что $\mu=0$. Если положить $S_{D}(m, n)=\sum_{(i, j) \in D \cap \Pi(m, n)} X(i, j)$ для произвольного множества $D \subset \mathrm{N}^{2}$ и считать, что $\sum_{(i, j) \in \varnothing} X(i, j)=0$, то

$$
S(m, n)=S_{A}(m, n)+S_{B}(m, n)+S_{C}(m, n)
$$

для всех $m \geqslant 1$ и $n \geqslant 1$, где $B$ и $C$ определены в лемме 1 . Усиленный закон больших чисел для сумм $S_{B}$ и $S_{C}$ вытекает из теоремы Колмогорова и условия (1.5) (напомним, что $\mu=0)$. Действительно, оба семейства множеств $\{B \cap \Pi(m, n) ;(m, n) \in A\}$ и $\{C \cap \Pi(m, n) ;(m, n) \in A\}$ являются линейно упорядоченными в силу леммы 1 . В частности, это означает, что оба семейства случайных величин $\left\{S_{B}(m, n) ;(m, n) \in A\right\}$ и $\left\{S_{C}(m, n) ;(m, n) \in A\right\}$ можно рассматривать как подпоследовательности $\left\{S_{m_{k}}\right\}$ и $\left\{S_{n_{k}}\right\}$ обычной последовательности сумм $\left\{S_{k}, k \geqslant 1\right\}$ независимых одинаково распределенных случайных величин $\left\{X_{i}, i \geqslant 1\right\}$, имеющих функцию распределения $F$. Заметим, что для сумм $S_{C}$ соответствуюшая подпоследовательность отвечает индексам $n_{k}=k(k+1) / 2$. Следовательно,

$$
\lim _{A} \frac{S_{B}(m, n)}{m n}=0, \quad \lim _{A} \frac{S_{C}(m, n)}{m n}=0 \quad \text { п.н., }
$$

поскольку $\operatorname{card}(B \cap \Pi(m, n)) \leqslant m n$ и $\operatorname{card}(C \cap \Pi(m, n)) \leqslant m n$.

Рассмотрим теперь суммы $S_{A}$. Определим усеченные случайные величины

и их частичные суммы

$$
Y(i, j)= \begin{cases}0, & (i, j) \notin A, \\ X(i, j) \mathbb{I}_{\{|X(i, j)|<i j\}}, & (i, j) \in A\end{cases}
$$

$$
T(m, n)=\sum_{i=1}^{m} \sum_{j=1}^{n} Y(i, j) .
$$

Заметим, что случайные величины $\left\{Y(i, j) ;(i, j) \in \mathbf{N}^{2}\right\}$ независимы и ограничены, в частности, они имеют второй момент. Более того,

$$
\sum_{m=1}^{\infty} \sum_{n=1}^{\infty} \frac{\mathrm{D} Y(m, n)}{(m n)^{2}}<\infty
$$

согласно лемме 3, которую мы докажем ниже. Последнее соотношение и аналог теоремы Колмогорова для кратных сумм независимых неодинаково распределенных случайных величин (см. работу [4]) влекут

$$
\mathbf{P}\{|T(m, n)-\mathbf{E} T(m, n)| \geqslant \varepsilon m n \text { б.ч. }\}=0 \quad \text { для всех } \varepsilon>0 .
$$

Поскольку $\mu=0$, то

$$
\mathbf{E} Y(i, j)= \begin{cases}0, & (i, j) \notin A, \\ -\int_{|X| \geqslant i j} X d \mathbf{P}, & (i, j) \in A .\end{cases}
$$

Следовательно, при любом $\varepsilon>0$

неравенство $|\mathbf{E} T(m, n)| \geqslant \varepsilon m n$ выполняется конечное число раз.

Действительно, зафиксируем $\delta>0$. Тогда неравенство $\int_{|X| \geqslant i j}|X| d \mathbf{P} \geqslant \delta$ выполняется только для конечного числа пар $(i, j)$. Если количество таких пар обозначить через $l(\delta)$, то

$$
\frac{|\mathbf{E} T(m, n)|}{m n} \leqslant \frac{1}{m n} \sum_{i=1}^{m} \sum_{j=1}^{n} \int_{|X| \geqslant i j}|X| d \mathbf{P} \leqslant \delta+\frac{l(\delta) \mathbf{E}|X|}{m n},
$$

что и доказывает (2.12).

Чтобы закончить доказательство достаточности, заметим, что

$$
\sum_{(i, j) \in A} \mathbf{P}\{X(i, j) \neq Y(i, j)\}=\sum_{(i, j) \in A} \mathbf{P}\{|X| \geqslant i j\}<\infty
$$


в силу условия (1.8). Применяя лемму Бореля-Кантелли для независимых событий, показываем, что с вероятностью 1 происходит только конечное число событий $\{\omega: X(i, j) \neq Y(i, j)\}$. Это означает, что с вероятностью 1 суммы $S_{A}(m, n)$ и $T(m, n)$ отличаются только на конечное число слагаемых. Объединяя этот результат с $(2.11)$ и (2.12), получаем

$$
\lim _{A} \frac{S_{A}(m, n)}{m n}=0 \text { п.н., }
$$

откуда и вытекает (2.3) ввиду (2.10). Теорема 1 доказана.

Докажем наконец лемму 3, которая использовалась в доказательстве теоремы 1. Заметим, что предположение о монотонности функции $f$ в лемме не обязательно, хотя оно необходимо в доказательстве теоремы 1.

Лемма 3. Пусть $X$ - случайная величина с конечньи математическим ожиданием. Пусть функиия $f$ удовлетворяет условию (2.1), а множество А определено согласно (2.2). Если выполнено условие (1.8), то

$$
\sum_{(i, j) \in A} \frac{1}{(i j)^{2}} \int_{\{\omega:|X(\omega)|<i j\}} X^{2} d \mathbf{P}<\infty
$$

Более того,

$$
\sum_{(i, j) \in A} \frac{1}{(i j)^{2}} \int_{\{\omega:|X(\omega)|<i j\}} X^{2} d \mathbf{P} \leqslant 2 \sum_{(i, j) \in A} \mathbf{P}\{|X| \geqslant i j\}+6 \mathbf{E}|X|+4 \mathbf{E}|X|^{1 / 2}+4 .
$$

Д ок аз а т ельст в о. Положим $p_{k}=\int_{k-1 \leqslant|X|<k} d \mathrm{P}$ для $k \geqslant 1$ и $R_{1}=$ $\sum_{(i, j) \in A} \mathbf{P}\{|X| \geqslant i j\}$. Ясно, что

$$
\begin{aligned}
R_{1} & =\sum_{(i, j) \in A} \sum_{k: k \geqslant i j} p_{k+1} \geqslant \sum_{(i, j) \in A} \sum_{k: i j \leqslant k \leqslant i f(i)} p_{k+1} \\
& =\sum_{i=1}^{\infty} \sum_{j: i \leqslant j \leqslant f(i)} \sum_{k: i j \leqslant k \leqslant i f(i)} p_{k+1}=\sum_{i=1}^{\infty} \sum_{k: i^{2} \leqslant k \leqslant i f(i)} \sum_{j: i \leqslant j \leqslant k / i} p_{k+1} \\
& =\sum_{i=1}^{\infty} \sum_{k: i^{2} \leqslant k \leqslant i f(i)} p_{k+1}\left(\left[\frac{k}{i}\right]-i+1\right) \geqslant \sum_{i=1}^{\infty} \sum_{k: i^{2} \leqslant k \leqslant i f(i)} p_{k+1}\left(\frac{k}{i}-i\right) \\
& =\sum_{i=1}^{\infty} \sum_{k: i^{2} \leqslant k \leqslant i f(i)} \frac{k}{i} p_{k+1}-\sum_{i=1}^{\infty} \sum_{k: i^{2} \leqslant k \leqslant i f(i)} i p_{k+1} .
\end{aligned}
$$

Второе выражение в правой части не превосходит $\mathbf{E}|X|$. Действительно,

$\sum_{i=1}^{\infty} \sum_{k: i^{2} \leqslant k \leqslant i f(i)} i p_{k+1} \leqslant \sum_{i=1}^{\infty} \sum_{k: k \geqslant i^{2}} i p_{k+1}=\sum_{k=1}^{\infty} p_{k+1} \sum_{i: i^{2} \leqslant k} i \leqslant \sum_{k=1}^{\infty} k \int_{k \leqslant|X| k+1} d \mathbf{P} \leqslant \mathbf{E}|X|$.

Определим теперь последовательность $\left\{\lambda_{k}, k \geqslant 1\right\}$ следующим образом:

$$
\lambda_{k}=\sum_{i: i^{2} \leqslant k, i f(i) \geqslant k} \frac{1}{i} \text {. }
$$

Тогда

$$
R_{1}+\mathbf{E}|X| \geqslant \sum_{k=1}^{\infty} k \lambda_{k} p_{k+1}
$$

Положим

$$
\sigma_{k}^{2}=\int_{k-1 \leqslant|X|<k} X^{2} d \mathbf{P}, \quad R_{2}=\sum_{(i, j) \in A} \frac{1}{(i j)^{2}} \int_{\{\omega:|X(\omega)|<i j\}} X^{2} d \mathbf{P} .
$$

Ясно, что

$$
R_{2}=\sum_{(i, j) \in A} \frac{1}{(i j)^{2}} \sum_{k=1}^{i j} \sigma_{k}^{2} \leqslant \sum_{(i, j) \in A} \frac{1}{(i j)^{2}} \sum_{k=1}^{i^{2}} \sigma_{k}^{2}+\sum_{(i, j) \in A} \frac{1}{(i j)^{2}} \sum_{k=i^{2}}^{i j} \sigma_{k}^{2} .
$$


Слагаемые в правой части обозначим через $R_{21}$ и $R_{22}$ соответственно. Первое из них не превосходит $4 \mathrm{E}|X|$. Действительно,

$$
\begin{aligned}
R_{21} & =\sum_{i=1}^{\infty} \sum_{j: i \leqslant j \leqslant f(i)} \sum_{k=1}^{i^{2}} \frac{\sigma_{k}^{2}}{(i j)^{2}}=\sum_{i=1}^{\infty} \sum_{k=1}^{i^{2}} \frac{\sigma_{k}^{2}}{i^{2}} \sum_{j: i \leqslant j \leqslant f(i)} \frac{1}{j^{2}} \leqslant \sum_{i=1}^{\infty} \sum_{k=1}^{i^{2}} \frac{\sigma_{k}^{2}}{i^{2}} \sum_{j=i}^{\infty} \frac{1}{j^{2}} \\
& \leqslant 2 \sum_{i=1}^{\infty} \sum_{k=1}^{i^{2}} \frac{\sigma_{k}^{2}}{i^{3}}=2 \sum_{k=1}^{\infty} \sigma_{k}^{2} \sum_{i: i^{2} \geqslant k} \frac{1}{i^{3}} \leqslant 4 \sum_{k=1}^{\infty} \frac{\sigma_{k}^{2}}{k} \leqslant 4 \mathbf{E}|X| .
\end{aligned}
$$

Слагаемое $R_{22}$ можно оценить следующим образом:

$$
\begin{aligned}
R_{22} & \leqslant \sum_{i=1}^{\infty} \sum_{j: i \leqslant j \leqslant f(i)} \frac{1}{(i j)^{2}} \sum_{k: i^{2} \leqslant k \leqslant i j} \sigma_{k}^{2} \leqslant \sum_{i=1}^{\infty} \sum_{k: i^{2} \leqslant k \leqslant i f(i)} \frac{\sigma_{k}^{2}}{i^{2}} \sum_{j: k / i \leqslant j \leqslant f(i)} \frac{1}{j^{2}} \\
& \leqslant 2 \sum_{i=1}^{\infty} \sum_{k: i^{2} \leqslant k \leqslant i f(i)} \frac{\sigma_{k}^{2}}{i k} \leqslant 2 \sum_{k=1}^{\infty} k p_{k} \lambda_{k} .
\end{aligned}
$$

Собирая вместе оценки для $R_{21}$ и $R_{22}$, получаем

$$
R_{2} \leqslant 4 \mathbf{E}|X|+2 \sum_{k=1}^{\infty} k p_{k} \lambda_{k}
$$

Чтобы закончить доказательство леммы, необходимо сравнить ряды $\sum_{k=1}^{\infty} k \lambda_{k} p_{k+1}$ и $\sum_{k=1}^{\infty} k \lambda_{k} p_{k}$. Положим $\lambda_{k}^{\prime}=\sum_{i: i^{2} \leqslant k+1, i f(i) \geqslant k} i^{-1}$. Понятно, что

Следовательно,

$$
\lambda_{k}^{\prime}-\lambda_{k}= \begin{cases}m^{-1}, & \text { если } k+1=m^{2} \text { для некоторого } m \geqslant 1, \\ 0 & \text { в ином случае. }\end{cases}
$$

$$
\sum_{k=1}^{\infty}(k+1) p_{k+1}\left(\lambda_{k}^{\prime}-\lambda_{k}\right)=\sum_{m=1}^{\infty} m^{2} p_{m^{2}} \frac{1}{m} \leqslant \mathbf{E}|X|^{1 / 2}+1 .
$$

С другой стороны, $\lambda_{k}^{\prime} \geqslant \lambda_{k+1}$ и поэтому

$$
\sum_{k=1}^{\infty}(k+1) p_{k+1} \lambda_{k+1} \leqslant \sum_{k=1}^{\infty}(k+1) p_{k+1} \lambda_{k}+\sum_{k=1}^{\infty}(k+1) p_{k+1}\left(\lambda_{k}^{\prime}-\lambda_{k}\right)
$$

откуда $\sum_{k=1}^{\infty} k p_{k} \lambda_{k} \leqslant 1+\sum_{k=1}^{\infty} k p_{k+1} \lambda_{k}+\sum_{k=1}^{\infty} p_{k+1} \lambda_{k}+\mathbf{E}|X|^{1 / 2}+1$. Наконец, $\lambda_{k} \leqslant \sqrt{k}$ и поэтому $\sum_{k=1}^{\infty} p_{k+1} \lambda_{k} \leqslant \mathbf{E}|X|^{1 / 2}$, откуда

$$
\sum_{k=1}^{\infty} k p_{k} \lambda_{k} \leqslant 2+\sum_{k=1}^{\infty} k p_{k+1} \lambda_{k}+2 \mathbf{E}|X|^{1 / 2}
$$

Объединяя эту оценку с (2.15) и (2.16), получаем (2.14). Лемма 3 доказана.

\section{3. Заключительные замечания и примеры.}

3 а м е ч а н и е 2. Теорему 1 легко обобщить на случай, рассмотренный в работе [5], где границы $A$ сформированы двумя функциями, т.е. $A=\left\{(i, j): g_{1}(i) \leqslant\right.$ $\left.j \leqslant g_{2}(i)\right\}$. Следует также отметить, что условие $(1.7)$, необходимое для метода работы [5], не предполагается в следуюшем результате.

Следствие 1. Пусть $g_{1}$ и $g_{2}$ - две неубьваюиие функиии, которые удовлетворяют условию (1.6), а $A=\left\{(i, j): g_{1}(i) \leqslant j \leqslant g_{2}(i)\right\}$. Усиленный закон больших чисел (2.3) эквивалентен условиям (1.5) и (1.8).

3 а м е ч а н и е 3. Метод, использованный в доказательстве теоремы 1, можно применить и в случае кратных сумм высших размерностей (т.е. для $d \geqslant 3$ ).

Ниже приведены несколько примеров для случая $g_{2}(x)=x$. Всюду ниже в формулировках мы используем последовательность $T_{n}, n \geqslant 1$, и функцию $T(x)$, определенные в замечании 1 , а также предполагаем, что $\mathbf{E} X=0$.

П р и м е р 1. Пусть $g_{1}(x)=1$ для всех $x \geqslant 1$. Из «грубой» оценки в проблеме множителей Цирихле вытекает, что $T_{n} \asymp n \ln n$, и поэтому условие (1.8) эквивалентно 
$\mathrm{E}|X| \ln ^{+}|X|<\infty$. Используя симметрию, мы выводим отсюда усиленный закон больших чисел Смайза [6].

П р и м е р 2. Пусть $0<\theta<1$ и $g_{1}(x)=\theta x$. Тогда $T_{n} \asymp n$ и мы получаем усиленный закон больших чисел Гута [3].

П р и м е р 3. Пусть $1>\delta>0$ и $g_{1}(x)=x^{1-\delta}$. Тогда $T_{n} \asymp n \ln n$ и поэтому усиленный закон больших чисел на $A=\left\{(i, j): i^{1-\delta} \leqslant j \leqslant i\right\}$ эквивалентен условию $\mathbf{E}|X| \ln ^{+}|X|<\infty$.

П р и м е р 4. Пусть $\delta>0$ и $g_{1}(x)=x /(\ln x)^{\delta}$. Тогда $T_{n} \asymp n \ln \ln n$ и усиленный закон больших чисел на $A=\left\{(i, j): i /(\ln i)^{\delta} \leqslant j \leqslant i\right\}$ эквивалентен условию $\mathrm{E}|X| \ln ^{+} \ln ^{+}|X|<\infty$.

П р и м е р 5 . Пусть $1>\delta \geqslant 0$ и $g_{1}(x)=x \exp \left\{-(\ln x)^{\delta}\right\}$. Тогда $T_{n} \asymp n(\ln n)^{\delta}$ и усиленный закон больших чисел на $A=\left\{(i, j): g_{1}(i) \leqslant j \leqslant i\right\}$ эквивалентен $\mathbf{E}|X|\left(\ln ^{+}|X|\right)^{\delta}<\infty$.

\section{СПИСОК ЛИТЕРАТУРЫ}

1. Baum L. E., Katz M. Convergence rates in the law of large numbers. - Trans. Amer. Math. Soc., 1965 , v. 120 , p. 108-123.

2. Dunford $N$. An individual ergodic theorem for noncommutative transformations. Acta Sci. Math. Szeged, 1951, v. 14, p. 1-4.

3. Gut $A$. Strong laws for independent identically distributed random variables indexed by a sector. - Ann. Probab., 1983, v. 11, № 3, p. 569-577.

4. Клесов О.И. Неравенство Гаека-Реньи для случайных полей и усиленный закон больших чисел. - Теория вероятн. матем. статист., 1980, т. 22, с. 58-66.

5. Клесов О.И., Рьхлик 3. Усиленный закон больших чисел на частично упорядоченных множествах. - Теория вероятн. матем. статист., 1998, т. 58, с. 31-37.

6. Smythe R.T. Strong laws of large numbers for $r$-dimensional arrays of random variables. - Ann. Probab., 1973, v. 1, № 1, p. 164-170.

7. Wiener N. The ergodic theorem. - Duke Math. J. 1939, v. 5, p. 1-18.

8. Zygmund $A$. An individual ergodic theorem for noncommutative transformations. Acta Sci. Math. Szeged., 1951, v. 14, p. 103-110.

Поступила в редакцию 18.III. 2004

Исправленный вариант 11.VII. 2007

(C) $2007 \mathrm{r}$.

ПРОХОРОВ Ю. В.", ХОХЛОВ В. И.*

\section{О МНОГОЧЛЕНАХ ОТ КОМПОНЕНТ ГАУССОВСКИХ СЛУЧАЙНЫХ ВЕКТОРОВ}

В настоящей статье уточняются и распространяются на многомерный случай результаты, полученные в [1] для одномерного случая.

Ключевые слова и фразы: многочлены от случайных величин, гауссовские векторы, многочлены Эрмита, формула Мелера

Напомним одну из теорем работы [1]. Пусть $Q(x)-$ многочлен степени $n \geqslant 2$, $Z$ - случайная величина, имеющая нормальное распределение вероятностей с параметрами 0 и 1 (т.е. стандартное нормальное распределение) и $Y=Q(Z)$.

Допустим $\mathbf{E} Y=0$.

* Математический институт им. В. А. Стеклова РАН, ул. Губкина, 8, 119991 Москва, ГСП-1, Россия; e-mail: tvp@tvp.ru 